\title{
GRB 130603B: No Compelling Evidence for Neutron Star Merger
}

\author{
Shlomo Dado and Arnon Dar \\ Physics Department, Technion, 32000 Haifa, Israel \\ Correspondence should be addressed to Arnon Dar; arnon@physics.technion.ac.il \\ Received 22 September 2014; Accepted 3 February 2015 \\ Academic Editor: WeiKang Zheng
}

Copyright (c) 2015 S. Dado and A. Dar. This is an open access article distributed under the Creative Commons Attribution License, which permits unrestricted use, distribution, and reproduction in any medium, provided the original work is properly cited.

\begin{abstract}
The near infrared (NIR) flare/rebrightening in the afterglow of the short hard gamma ray burst (SHB) 130603B measured with the Hubble Space Telescope (HST) and an alleged late-time X-ray excess were interpreted as possible evidence of a neutron star merger origin of SHBs. However, the X-ray afterglow that was measured with the Swift XRT and Newton XMM has the canonical behaviour of a synchrotron afterglow produced by a highly relativistic jet. The H-band flux observed with HST 9.41 days after burst is that expected from the measured late-time X-ray afterglow. The late-time flare/rebrightening of the NIR-optical afterglow of SHB 130603B could have been produced also by jet collision with an interstellar density bump. Moreover, SHB plus a kilonova can be produced also by the collapse of a compact star (neutron star, strange star, or quark star) to a more compact object due to cooling, loss of angular momentum, or mass accretion.
\end{abstract}

\section{Introduction}

Stripped envelope supernova explosions and neutron star mergers in close binaries were originally suggested by Goodman et al. [1] as possible sources of cosmological gamma ray bursts. However, their proposed underlying mechanism-a spherical fireball produced by neutrino-antineutrino annihilation into electron positron pairs beyond the surface of the collapsing/merging star-turned out not to be powerful enough to produce GRBs observable at very large cosmological distances as indicated from analysis of the first 153 GRBs observed with the Burst and Transient Source Experiment aboard the Compton Gamma Ray Observatory [2], which was launched in 1991. Consequently, Shaviv and Dar proposed [3] that highly relativistic jets of ordinary matter are probably ejected in such events and produce narrowly collimated GRBs by inverse Compton scattering of circumstellar light. They also suggested that short GRBs may also be produced by highly relativistic jets ejected in the phase transition of compact stars, such as neutron stars, strange stars, and quark stars, into more compact objects due to mass accretion or to cooling and loss of angular momentum via winds and radiation. After the discovery of GRB afterglows, Dar [4] proposed that they are highly beamed synchrotron radiation emitted by these highly relativistic jets in their collision with the interstellar matter.

By now, there is convincing evidence that long duration GRBs and their afterglows are produced mostly by highly relativistic jets launched in stripped envelope supernova explosions (mainly of type Ic), but, despite the enormous observational efforts, the origin of short duration GRBs remains unknown. In fact, the circumstantial evidence that has been claimed to link short hard GRBs (SHBs) with neutron star merger in close binaries, such as their location in both spiral and elliptical galaxies $[5,6]$ and the distribution of their location offsets relative to the center of their host galaxies, which extends to a distance of $100 \mathrm{kpc}$ [6] and beyond (e.g., SHB 080503 with the lack of a coincident host galaxy down to $28.5 \mathrm{mag}$ in deep Hubble Space Telescope imaging [7]), actually favours a phase transition in a single compact star [8] with a large natal kick velocity over merging neutron stars in neutron star binaries [1] whose velocities are much smaller [9].

A more direct observational evidence that SHBs are produced by neutron stars merger was proposed by $\mathrm{Li}$ and Paczynski [10]. These authors suggested that neutron star mergers may create significant quantities of neutronrich radioactive nuclei whose decay should result in a faint 
transient in the days following the burst, a so-called kilonova or macronova.

Recently, the broad band afterglow of the SHB 130603B (Melandri et al. [11] and Golenetskii et al. [12]) that was measured with the Swift X-ray telescope (XRT), Newton XMM, HST, and ground-based optical and radio telescopes was interpreted by Tanvir et al. [13, 14], Berger et al. [15], and Fong et al. [16] as evidence supporting a neutron star merger origin of SHB 130603B. However, in this paper we show that the X-ray afterglow of SHB 130603B, which was measured with Swift XRT (Swift-XRT GRB light-curve repository [17]) and Newton XMM [16], had the canonical behaviour of a synchrotron afterglow produced by a highly relativistic jet propagating in a normal interstellar environment, as predicted by the cannonball model of GRBs [8, 18-20] long before its empirical discovery by Nousek et al. with Swift [21]. This canonical X-ray afterglow does not have a "mysterious late-time X-ray excess" as claimed in [16], and the flux observed in the NIR H-band with HST 9.6 days after burst $[13,14]$ is that expected from the measured late-time $\mathrm{X}$-ray afterglow. Moreover, a fast decline of a late-time afterglow followed by a rebrightening/flare in the NIR and optical afterglow of a GRB can be produced by a jet colliding with a density bump in the interstellar medium [8], as was observed in several long duration GRBs, such as $030329[22,23]$ and 070311 [24], and SHBs such as 050724 [25] and 080503 [26]. The host galaxy of SHB $130603 \mathrm{~B}$ at redshift $z=0.356$ [7], as seen in high-resolution HST imaging, is a perturbed spiral galaxy due to interaction with another galaxy [27]. SHB 130603B was located in one of its tidally disrupted arms [27]. The interaction of the SHB jet with such a bumpy environment may have caused the flare/rebrightening in the NIR afterglow observed with the HST on day 9.41 $[13,14]$.

Furthermore, a late-time flare/rebrightening of a NIRoptical afterglow of SHB can be produced by either a jet collision with an interstellar density bump or a kilonova. However, SHB plus kilonova can be produced also by collapse of compact stars (neutron star, strange star, or quark star) to a more compact object due to cooling, loss of angular momentum, or mass accretion $[3,8,28]$. The distribution of pulsar velocities has a high velocity component due to single pulsars and a lower velocity component from pulsars in binaries and isolated millisecond pulsars [9]. Hence, single compact stars are more likely than neutron star binaries (neutron stars, neutron star-black hole, and neutron starwhite dwarf binaries) to be found at the large observed offsets of several SHBs from the center/disk of their host galaxies or at far away distances where no nearby host candidate was found in very deep searches.

Finally, the star formation within the host, location of SHB $130603 B$ on top of the tidally disrupted arm, strong absorption features, and large line of sight extinction that were observed indicate that the GRB progenitor was probably not far from its birth place [27], untypical of the rather long mean lifetime before neutron star merger due to gravitational wave emission estimated for the known neutron star binaries in our galaxy (see, e.g., [9] for a recent review).

\section{The X-Ray Afterglow of SHB 130603B}

The conclusion of Fong et al. [16] that the X-ray afterglow of SHB 130603B shows "a mysterious late-time X-ray excess" was based on a standard fireball model analysis of its X-ray afterglow. The standard fireball model, however, predicts that the temporal index $\alpha$ of the afterglow of a conical jet that is parametrized as a smoothly broken power law, $F_{v} \propto t^{-\alpha} v^{-\beta}$, increases by $\Delta \alpha_{\mathrm{X}}=0.75$ across the jet break, independent of the spectral index $\beta_{\mathrm{X}}$ of the afterglow [20]. The X-ray temporal indices $\alpha_{\mathrm{X}}=0.35 \pm 0.08$ and $\alpha_{\mathrm{X}}=1.61 \pm 0.08$ before and after the break, respectively, that were reported in the Swift-XRT GRB Catalogue [17] yield $\Delta \alpha_{\mathrm{X}}=1.26 \pm 0.11$ $\left(\alpha_{\mathrm{X}}=1.75 \pm 0.08\right.$ for the combined data of Swift XRT and Newton XMM yields $\Delta \alpha_{\mathrm{X}}=1.40 \pm 0.11$ ), which is at odds with $\Delta \alpha_{\mathrm{X}} \approx 0.75$ expected in the conical fireball model.

In contrast, the X-ray afterglow of SHB 130603B that was measured with Swift XRT [17] and Newton XMM [16] has the canonical behaviour of a normal synchrotron afterglow produced by a highly relativistic jet propagating in a normal interstellar environment of its host galaxy as predicted by the CB model [18] long before the launch of Swift and its empirical discovery by Nousek et al. [21]. It consists of an early plateau phase that follows the fast decline phase of the prompt emission and breaks smoothly into a late-time $(t \gg$ $t_{b}$ ) power law decline with a power law index that satisfies the cannonball (CB) model closure relation $[19,20]$

$$
\alpha_{X}=\beta_{X}+\frac{1}{2}=\Gamma_{X}-\frac{1}{2},
$$

independent of the prebreak power law index, where $\Gamma_{X}$ is the photon spectral index of the X-ray afterglow. Using the value $\beta_{\mathrm{X}}=1.15 \pm 0.11$, which was obtained by de Ugarte Postigo et al. [27] from the Swift-XRT data, the CB model closure relation yields $\alpha_{\mathrm{X}}=1.65 \pm 0.11$. This value is consistent within errors with the postbreak value $\alpha_{\mathrm{X}}=1.61 \pm 0.08$ reported for SHB 130603B in the Swift-XRT GRB Catalogue [17].

In the $\mathrm{CB}$ model, the canonical light curve of the $\mathrm{X}$ ray afterglow depends only on three parameters [19]: the product $\gamma \theta$ of the bulk motion Lorentz factor of the jet and the viewing angle relative to the direction of motion of the jet, the jet deceleration parameter $t_{0}$, and the spectral index $p_{e}$ of the Fermi accelerated electrons in the jet that satisfies $p_{e}=2 \beta_{\mathrm{X}}$. CB model fit to the light curve of the $0.3-10 \mathrm{keV}$ X-ray afterglow of SHB 130603B, which was measured with Swift XRT [17] and with Newton XMM assuming the spectral index that was measured with Swift, is shown in Figure 1. The best fit value $p_{e}=2.37$ yields $\beta_{\mathrm{X}}=p_{e} / 2=1.18$, which is consistent with the late-time photon index $\beta_{\mathrm{X}}+1=\Gamma_{\mathrm{X}}=$ $2.21 \pm 0.18$ reported in the Swift-XRT GRB Catalogue [17]. The two other best fit parameters, $\gamma \theta=0.55$ and $t_{0}=878 \mathrm{~s}$, yield a deceleration break (so-called jet break) at $t_{b} \approx 1500 \mathrm{~s}$.

Thus, we conclude that there is no evidence for a "mysterious late-time X-ray excess" that was claimed in [16] and was explained by a magnetar contribution to the afterglow emission of SHB 130603B [29, 30]. 


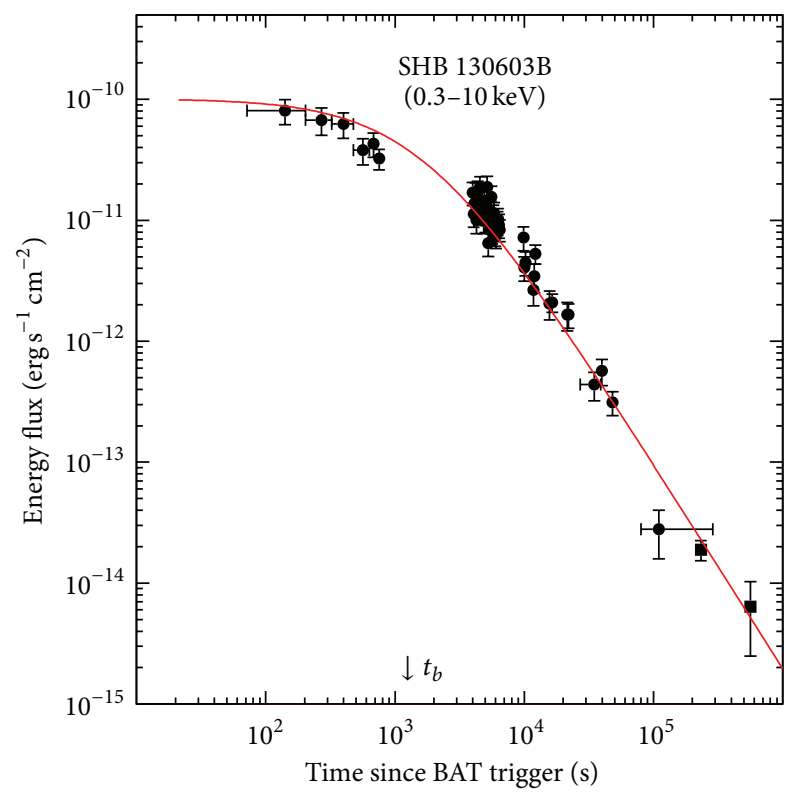

FIGURE 1: Comparison between the light curve of the X-ray afterglow of SHB 130603B, which was measured with Swift XRT [17] and with Newton XMM [16] assuming the spectral index $\beta_{\mathrm{X}}=1.15$ that was measured with Swift, and a CB model fit.

\section{The Near Infrared-Optical Afterglow}

The conclusion that the NIR-optical afterglow of SHB $130603 \mathrm{~B}$ provides possible evidence of a macronova/kilonova was based on a rebrightening of the NIR afterglow observed with the Hubble Space Telescope (HST) in the H-band on day 9.41, which is well above that extrapolated from the fast decline of the optical afterglow in the $r$ band during the first day after the break around $0.3 \mathrm{~d}[15,16]$. However, in the CB model, when the spectral index of the late-time NIR and optical bands is above the spectral break, $\beta_{\mathrm{H}} \approx \beta_{\mathrm{X}}$ and consequently $\alpha_{\mathrm{H}}=\beta_{\mathrm{H}}+1 / 2 \approx \alpha_{\mathrm{X}}$. Using the groundbased JK-band observations extrapolated to the H-band [16] and the HST H-band measurement, we obtained the fact that $\alpha_{\mathrm{H}}=1.69 \pm 0.08$ in the time interval $0.61-9.41 \mathrm{~d}$, which is in agreement, within errors, with the power law index $\alpha_{\mathrm{X}}=1.68 \pm 0.08$ of the joint late-time Swift-XRT observations [17] and XMM Newton observations [16]. This is shown in Figure 2 where we compare the late-time $\mathrm{H}$-band spectral energy density (SED) of SHB 130603B [14, 15], corrected for line of sight extinction [16], and the SED expected from the $\mathrm{CB}$ model fit to the X-ray afterglow.

Moreover, a broken power law best fit to the unabsorbed late-time broad band NIR-optical and Swift X-ray spectrum by de Ugarte Postigo et al. [27] yielded $\beta=0.65 \pm 0.09$ below a break at $v_{b}=9.55 \times 10^{15} \mathrm{~Hz}$ and $\beta_{\mathrm{X}}=1.15 \pm 0.11$. Using $\lambda=$ 12.4 A for $1 \mathrm{keV}$ photons, $\lambda=16300 \mathrm{~A}$ for $H$-band photons and $\lambda_{\text {break }}=314 \mathrm{~A}$; the expected flux ratio of the $\mathrm{H}$ and $\mathrm{X}$-ray bands is $F_{\mathrm{H}} / F_{\mathrm{keV}} \approx 536 \pm 160$. This ratio is in good agreement within errors with the observed ratio $F_{\mathrm{H}} / F_{\mathrm{keV}}=623 \pm 160$ of the H-band flux measured with HST on day 9.41 after burst and the $1 \mathrm{keV} \mathrm{X}$-ray flux obtained by extrapolating the joint Swift-XRT and Newton XMM $1 \mathrm{keV}$ flux to day 9.41.

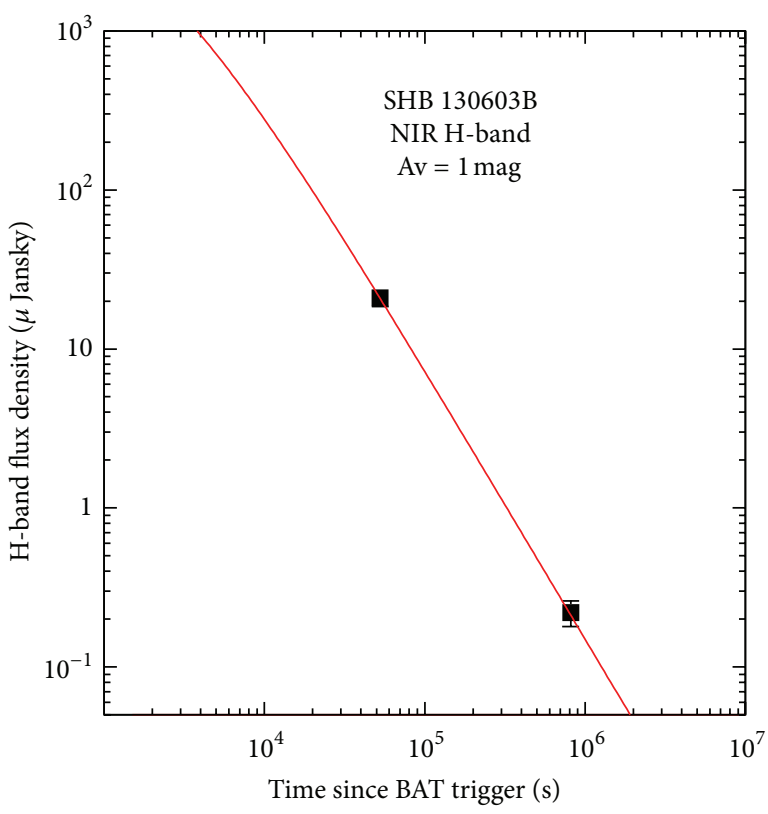

FIgure 2: Comparison between the observed late-time H-band spectral energy density $[14,15]$ of SHB 130603B, where the JK-band observations were extrapolated to the $\mathrm{H}$-band and corrected for line of sight extinction [16], and that expected from the CB model fit of the X-ray afterglow shown in Figure 1.

The highly relativistic jets of plasmoids (cannonballs) that produce GRBs can encounter a bumpy interstellar medium in the host galaxy. Also, the opacity along the line of sight to the jet in the host can vary significantly due to the "superluminal" motion of the line of sight to the jet in the host galaxy. The collision of a jet with an overdensity bump can produce chromatic rebrightening/flare in the NIR-optical afterglow (e.g., $[8,18])$ as was observed in the late-time optical afterglow of several long duration GRBs such as 030329 [22, 23] and 070311 [24] and of SHBs such as 050724 [25] and 080503 [26], while underdensity can cause a fast temporal decline of an afterglow $(\alpha>2)$, as observed in several GRBs (Swift-XRT GRB Catalogue [17]). Such density variations cause spectral and temporal variations in the afterglow, which otherwise has a smooth power law behaviour. After an overdensity or an underdensity, the late-time $\left(t \gg t_{b}\right)$ closure relation of the CB model is recovered when the column density as function of distance converges to that of the mean ISM density. This can explain both a fast decline of the NIR-optical afterglow of SHB 130603B after an overdensity followed by an underdensity and a recovery to the normal power law decline like that of the $\mathrm{X}$ ray afterglow.

\section{The Macronova-SHB Association}

SHBs may be produced by highly relativistic jets launched in the collapse of compact stars (neutron star, strange star, or quark star) to a more compact object due to loss of angular momentum, cooling, or mass accretion $[3,8,28]$. During neutron star mergers, or collapse of compact stars to more compact objects, the crust layers may be stripped 
off by very strong outgoing shocks. Neutrino-antineutrino annihilation into electron-positron pairs behind such blownoff layers perhaps can produce a fireball [1] which may accelerate the blown-off crust layers to velocities well above the escape velocity (Li and Paczynski [10]) although the neutrino luminosity, which is well below the "neutrino Eddington luminosity" [28], by itself cannot blow off the crust layers of a compact star.

It is unclear whether a robust r-process occurs in the macronova ejecta, or whether neutrinos drive the composition towards ${ }^{56} \mathrm{Ni}$ dominated composition (e.g., [29]). All together, the total mass of the ejecta, its composition, density, and velocity and their radial and angular distributions are highly uncertain, which makes the predicted signal from an associated macronova [10] very uncertain and unreliable for distinguishing between a phase transition in a single compact star and a merger of compact stars in binaries as the origin of SHBs.

\section{Conclusions}

Several explanations of the rebrightening of the NIR afterglow of SHB 130603B around 9.41 days after burst have been proposed. These include a macronova/kilonova produced by a neutron star merger in a close binary $[15,16]$, a millisecond magnetar produced in neutron star merger in close binaries $[30,31]$, and a late-time flare produced by collision of the SHB jet with an ISM density bump $[8,18]$.

SHBs plus a minisupernova/macronova/kilonova, however, are not unique to the neutron star merger scenario. They can be produced also in a phase transition/collapse of single compact stars (neutron star, strange star, or quark star) to a more compact object due to cooling, loss of angular momentum, or mass accretion.

The X-ray afterglow that was measured with the Swift XRT and Newton XMM has the expected canonical behaviour of a synchrotron afterglow produced by a highly relativistic jet. Its late-time behaviour does not provide an evidence for a millisecond magnetar as the power-source of the chromatic afterglow of SHB 130603B. The late-time H-band flux observed with HST 9.41 days after burst is that expected from an ordinary synchrotron radiation from a jet that produced the measured late-time X-ray afterglow.

Late-time flare/rebrightening of a NIR-optical afterglow of an SHB can be produced also by jet collision with an interstellar density bump, as seen in several GRBs. The host galaxy of SHB 130603B as seen in high-resolution HST imaging $[14,15]$ is a perturbed spiral galaxy due to interaction with another galaxy. The GRB was located in a tidally disrupted arm of its host galaxy [27]. The interaction of the GRB jet with such a bumpy environment could produce the flare/rebrightening of the NIR afterglow of the GRB observed with the HST 9.41 days after burst.

The star formation within the host, location of SHB 130603B on top of the tidally disrupted arm, strong absorption features, and large line of sight extinction that were observed indicate that the GRB progenitor was probably not far from its birth place [27], untypical to the usually very long lifetime before neutron star merger due to gravitational wave emission in the known neutron star binaries in our galaxy. Moreover, the failure to detect a host galaxy down to 28.5 mag in deep Hubble Space Telescope imaging searches in the case of, for example, SHB 080503 with a late-time flare/rebrightening [26] suggests a large natal kick velocity of its progenitor, unlikely for compact binaries, but often observed for isolated neutron stars/pulsars $[9,32]$.

Although radio data and spectroscopy of the late-time afterglow of short hard bursts can provide complementary information on the origin of SHBs [33], the true smoking gun for the neutron star merger in close binaries is the detection of gravitational waves. Unfortunately, this is unlikely to occur before the completion of the new generation of gravity-wave detectors, as the sensitivity of current detectors such as LIGO and Virgo is several orders of magnitude below what would be required to detect a merger at a distance similar to the nearest SHBs with known redshift.

\section{Conflict of Interests}

The authors declare that there is no conflict of interests regarding the publication of this paper.

\section{Acknowledgment}

The authors thank A. de Ugarte Postigo for useful comments.

\section{References}

[1] J. Goodman, A. Dar, and S. Nussinov, "Neutrino annihilation in type II supernovae," The Astrophysical Journal, vol. 314, pp. L7-L10, 1987.

[2] C. A. Meegan, G. J. Fishman, R. B. Wilson et al., "Spatial distribution of $\gamma$-ray bursts observed by BATSE," Nature, vol. 355, no. 6356, pp. 143-145, 1992.

[3] N. J. Shaviv and A. Dar, "Gamma-ray bursts from minijets," Astrophysical Journal Letters, vol. 447, no. 2, pp. 863-873, 1995.

[4] A. Dar, "Can fireball models explain gamma-ray bursts?" Astrophysical Journal Letters, vol. 500, no. 2, pp. L93-L96, 1998.

[5] E. Nakar, "Short-hard gamma-ray bursts," Physics Reports, vol. 442, no. 1-6, pp. 166-236, 2007.

[6] E. Berger, "Short-duration gamma-ray bursts," Annual Review of Astronomy and Astrophysics, vol. 51, pp. 43-105, 2014.

[7] D. A. Perley, B. D. Metzger, and J. Granot, "GRB 080503: implications of a naked short gamma-ray burst dominated by extended emission," The Astrophysical Journal, vol. 696, pp. 1871-1885, 2009.

[8] S. Dado, A. Dar, and A. de Rújula, "Short hard gamma-ray bursts and their afterglows," The Astrophysical Journal, vol. 693, pp. 311-328, 2009.

[9] D. R. Lorimer, "Binary and millisecond pulsars," Living Reviews in Relativity, vol. 11, article 8, 2008.

[10] L. X. Li and B. Paczynski, "Transient events from neutron star mergers," The Astrophysical Journal, vol. 507, no. 1, pp. L59-L62, 1998.

[11] A. Melandri, W. H. Baumgartner, and D. N. Burrows, "GRB 130603B: swift detection of a bright short burst," GCN Circulars Archive, \# 14735, 2013. 
[12] S. Golenetskii, R. Aptekar, and D. Frederiks, "Konus-wind observation of GRB 130603B," GCN Circulars Archive no. 14771, 2013.

[13] N. R. Tanvir, "GRB 130603B: HST limits on an underlying supernova," Gamma-Ray Coordination Network (GCN), Circular Archive, 14893, 2013, http://gcn.gsfc.nasa.gov/gcn/.

[14] N. R. Tanvir, A. J. Levan, A. S. Fruchter et al., "A "kilonova" associated with the short-duration $\gamma$-ray burst GRB 130603B," Nature, vol. 500, no. 7464, pp. 547-549, 2013.

[15] E. Berger, W. Fong, and R. Chornock, "An $r$-process kilonova associated with the short-hard GRB 130603B," The Astrophysical Journal Letters, vol. 774, no. 2, pp. L23-L26, 2013.

[16] W. Fong, E. Berger, and B. D. Metzger, "Short GRB 130603B: discovery of a jet break in the optical and radio afterglows, and a mysterious late-time X-ray excess," The Astrophysical Journal, vol. 780, pp. 118-126, 2014.

[17] P. A. Evans, A. P. Beardmore, K. L. Page et al., "Methods and results of an automatic analysis of a complete sample of Swift-XRT observations of GRBs," Monthly Notices of the Royal Astronomical Society, vol. 397, no. 3, pp. 1177-1201, 2009.

[18] S. Dado, A. Dar, and A. de Rújula, "On the optical and X-ray afterglows of gamma ray bursts," Astronomy and Astrophysics, vol. 388, no. 3, pp. 1079-1105, 2002.

[19] S. Dado, A. Dar, and A. de Rújula, "The diverse broadband light curves of swift gamma-ray bursts reproduced with the Cannonball model," The Astrophysical Journal, vol. 696, no. 1, pp. 994-1020, 2009.

[20] S. Dado and A. Dar, "Conical fireballs, cannonballs, and jet breaks in the afterglows of gamma ray bursts," Astronomy and Astrophysics, vol. 558, pp. A115-A121, 2013.

[21] J. A. Nousek, C. Kouveliotou, and D. Grupe, "Evidence for a canonical gamma-ray burst afterglow light curve in the Swift XRT Data," The Astrophysical Journal, vol. 642, no. 1, pp. 389400, 2006.

[22] Y. M. Lipkin, E. O. Ofek, A. Gal-Yam et al., "The detailed optical light curve of GRB 030329," The Astrophysical Journal, vol. 606, no. 1, pp. 381-394, 2004.

[23] T. Matheson, P. M. Garnavich, and K. Z. Stanek, "Photometry and spectroscopy of GRB 030329 and its sssociated supernova 2003dh: the first two months," The Astrophysical Journal, vol. 599, pp. 394-407, 2003.

[24] C. Guidorzi, S. D. Vergani, S. Sazonov et al., "GRB 070311: a direct link between the prompt emission and the afterglow," Astronomy and Astrophysics, vol. 474, pp. 793-805, 2007.

[25] D. Malesani, S. Covino, P. D’Avanzo et al., "Multicolor observations of the afterglow of the short/hard GRB 050724," Astronomy and Astrophysics, vol. 473, pp. 77-84, 2007.

[26] C. C. Thone, A. de Ugarte Postigo, J. Gorosabel, N. Tanvir, and J. P. U. Fynbo, "GRB 130603B: short GRB afterglow spectrum from GTC," GCN Circulars Archive, \# 14771, 2013.

[27] A. de Ugarte Postigo, C. C. Thöne, and A. Rowlinson, "Spectroscopy of the short-hard GRB 130603B: the host galaxy and environment of a compact object merger," Astronomy \& Astrophysics, vol. 563, pp. 62-68, 2013.

[28] A. Dar, B. Kozlovsky, S. Nussinov, and R. Ramaty, "Gamma-ray bursts and cosmic rays from accretion-induced collapse," The Astrophysical Journal, vol. 388, pp. 164-170, 1992.

[29] R. Surman, G. C. McLaughlin, M. Ruffert, H.-T. Janka, and W. R. Hix, " $r$-process nucleosynthesis in hot accretion disk flows from black hole-neutron star mergers," The Astrophysical Journal Letters, vol. 679, no. 2, pp. L117-L120, 2008.
[30] B. D. Metzger and A. L. Piro, "Optical and X-ray emission from stable millisecond magnetar formed from merger of binary neutron stars," Monthly Notices of the Astronomical Royal Society, vol. 439, pp. 3916-3930, 2014.

[31] Y. Fan, Y.-W. Yu, and D. Xu, "A supramassive magnetar central engine for GRB 130603B," The Astrophysical Journal, vol. 779, no. 2, pp. L25-L28, 2013.

[32] G. Hobbs, D. R. Lorimer, A. G. Lyne, and M. Kramer, "A statistical study of 233 pulsar proper motions," Monthly Notices of the Royal Astronomical Society, vol. 360, pp. 974-992, 2005.

[33] Z.-P. Jin, D. Xu, and Y.-Z. Fan, "Is the late near-infrared bump in short-hard GRB 130603B due to the Li-Paczynski kilonova?" The Astrophysical Journal, vol. 775, pp. L19-L22, 2013. 

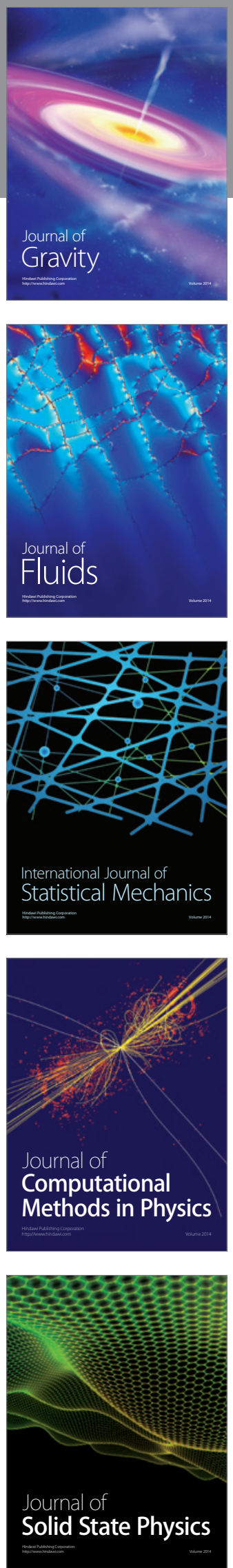

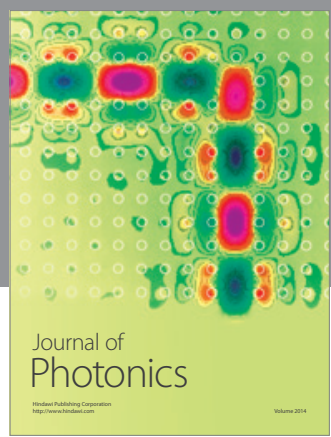

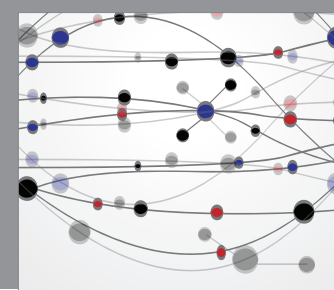

The Scientific World Journal

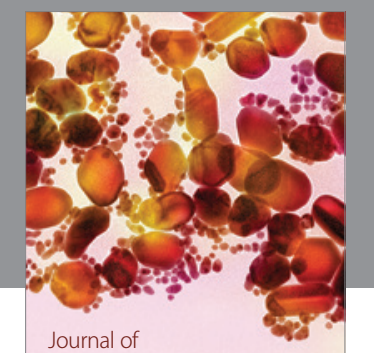

Soft Matter
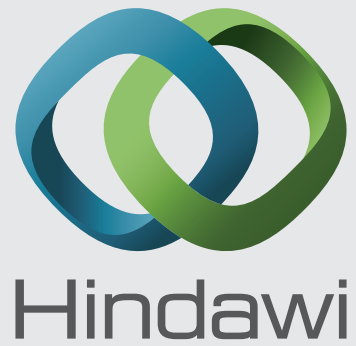

Submit your manuscripts at

http://www.hindawi.com
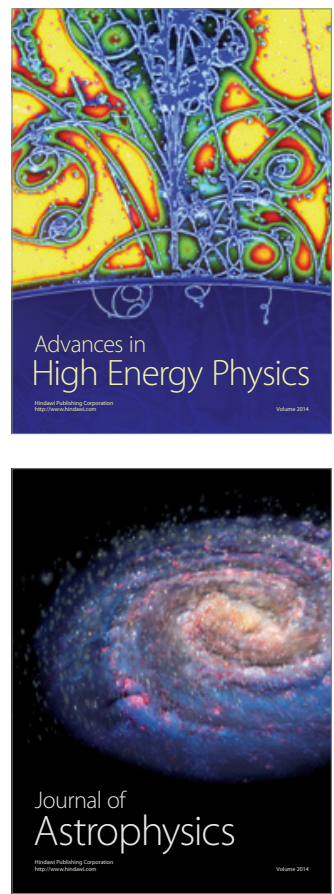
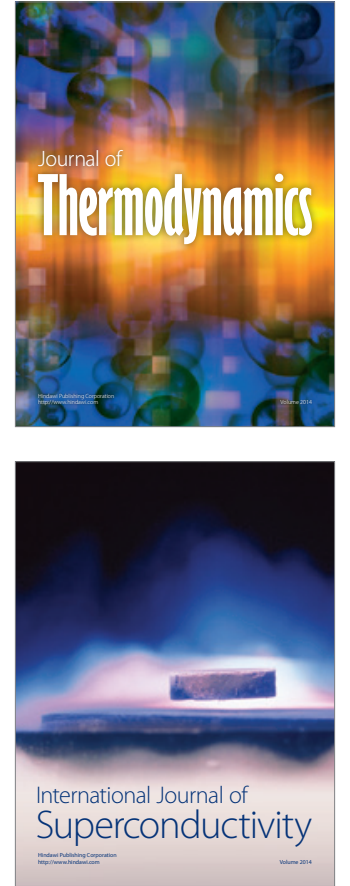
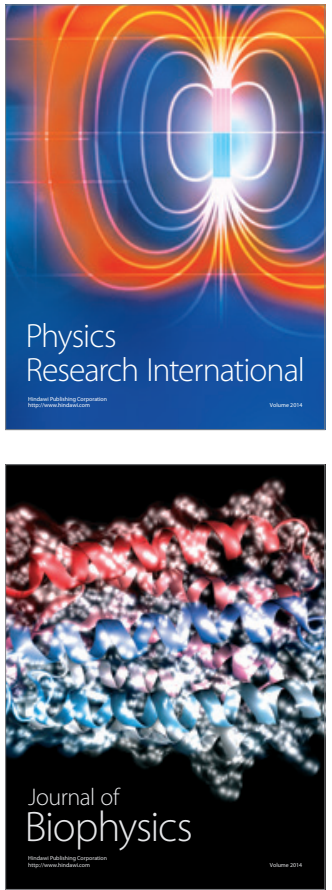
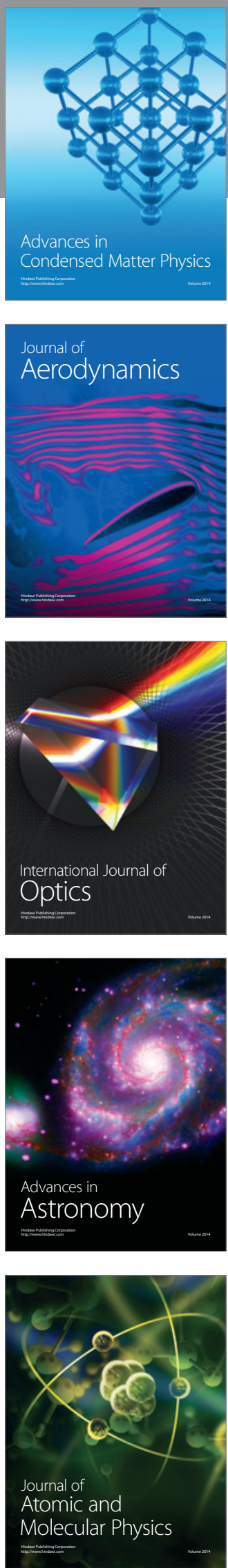